\title{
Miz-1 promotes the proliferation of esophageal cancer cells via suppression of p21 and release of p21-arrested cyclin D1
}

\author{
JUN LUO ${ }^{1,2}$, CHENG ZHANG ${ }^{1}$, CONGYI WANG ${ }^{4}$, LINJUN LI $^{1}$, CHUNHONG LI $^{3}$, \\ QIANG $\mathrm{LI}^{1}$, MIN ZHANG ${ }^{1}$ and QINGCHEN WU ${ }^{1}$ \\ ${ }^{1}$ Department of Cardiothoracic Surgery, The First Affiliated Hospital, Chongqing Medical University, Yuzhong, Chongqing; \\ ${ }^{2}$ Chongqing Key Laboratory of Molecular Oncology and Epigenetics, Yuzhong, Chongqing; ${ }^{3}$ Department of Oncology, \\ Suining Sichuan Center Hospital, Yuzhong, Sichuan; ${ }^{4}$ Department of Cardiothoracic Surgery, \\ People's Hospital of Changshou, Yuzhong, Chongqing, P.R. China
}

Received December 4, 2015; Accepted January 8, 2016

DOI: 10.3892/or.2016.4731

\begin{abstract}
Tumorigenesis results from various types of dysregulation of oncogenes and tumor suppressors that influence cellular proliferation, differentiation, apoptosis and senescence. The transcription factor Myc-interacting zinc finger protein 1 (Miz-1) can either activate or repress gene expression in concert with binding partners including the Myc oncoprotein in several types of tumors. Known target genes of these complexes encode the cyclin-dependent kinase inhibitors such as cdkn2b (p15) and cdkn1a (p21). In the present study, we showed that the silencing of Miz-1 expression, through shRNA in a lentiviral vector, influenced various biological processes in two types of esophageal carcinoma cell lines. Silencing of the expression of Miz-1 inhibited cell proliferation and promoted apoptosis in vitro. Loss of Miz-1 reduced the migration ability in esophageal carcinoma cells. High expression of p21 and downregulation of cyclin D1 accompanied the knockdown of Miz-1. Our data demonstrated that esophageal cancer has a cell cycle arrest pathway via Miz-1, p21 and cyclin D1.
\end{abstract}

\section{Introduction}

Proto-oncogenes of the Myc family play important roles in cellular growth, differentiation and cytometaplasia by encoding different oncogenic factors. c-Myc, a well-studied and critical Myc family member, is present in a variety of cancer cells including lung and breast cancer $(1,2)$. c-Myc also has a vital function in different cancers, including stomach, colon and bladder cancer $(3,4)$. Increasing numbers of target

Correspondence to: Professor Qingchen Wu, Department of Cardiothoracic Surgery, The First Affiliated Hospital, Chongqing Medical University, 1 Youyi Road, Yuzhong, Chongqing 400016, P.R. China

E-mail: qcwucq@163.com

Key words: Miz-1, esophageal cancer, cell cycle, signaling pathway, oncogene, p21 genes activated by c-Myc have been identified $(5,6)$. The c-Myc proto-oncogene encodes a transcription factor, Myc, which forms a heterodimer for its biological effects.

Myc-interacting zinc finger protein 1 (Miz-1; Zbtb17) is a zinc finger transcription factor that forms a complex with various oncoproteins such as Myc, Gfi-1 and Bcl-6 for its biological activity (7-9). There are three main mechanisms by which conjugation of Miz-1 and Myc plays a role in oncogenesis.

Firstly, the Myc/Miz-1 protein complex participates in the Hedgehog (Hh) signaling pathway. Miz-1 combines with Smo, the initiator of the Hh signaling pathway through subsequent downstream signaling factor Gli2, which then translocates into the nucleus its functional site (10). Ras driven tumorigenesis can be restrained by the Mule/Huwe1/Arf-BP1 pathway factors, via suppressing c-Myc/Miz-1-mediated regulation of p21 and p15 (11). Binding of Myc to Miz-1 suppresses TGF- $\beta$-dependent signaling via reducing CKI expression and cellular senescence $(12,13)$. Myc also inhibits expression of $\mathrm{p} 21^{\mathrm{CIP} 1}$ in response to DNA damage and to inducers of differentiation in cultured cells via Miz-1 $(14,15)$.

Secondly, Miz-1 associates with Myc on a number of target promoters, including two promoters of cell cycle inhibitors p21 and p15 (14). Similarly, CDKN1C (p57 ${ }^{\mathrm{KIP} 2}$ ) is repressed by Myc in a Miz-1-dependent manner $(12,16)$. Miz-1 can also act as a platform for transcription factors containing Bcl- 6 oncoprotein and Gli1 to repress cell cycle inhibitors. In addition, cell adhesion molecules, most notably integrins, also target the Miz-1/Myc complex (17). Moreover, Miz-1 was found to upregulate antiapoptotic Bcl-2 to stimulate IL-7 in a Miz-1deficient T lymphocyte model (18).

Thirdly, Myc/Miz-1 promotes tumor formation and development in many types of cancers. In skin carcinoma, studies have shown that Miz-1 inhibits the expression of tumorsuppressor protein $\mathrm{p} 21$, which may be related to ubiquitin ligating enzyme HectH9/Hunel $(11,19)$. During lymphomagenesis, the Myc/Miz-1 complex has a different effect on TGF- $\beta$-induced senescence (11). Similar effects have been observed for tumor formation in medulloblastoma. Likewise, Miz-1-deficient models show cancer repression (20). Most studies have focused on the Miz-1/Myc complex and cell cycle inhibitors such as p21 and p15. 
p21 is an inhibitor of CDK functioning downstream of p53 by two cellular pathways. One pathway is dependent on $\mathrm{p} 53$, the other is independent. The p21 protein can inhibit the activity of cyclin D1-CDK4 and cyclin E-CDK2, which further prevents the phosphorylation of $\mathrm{Rb}$ protein, resulting in cell cycle arrest in the G1 phase (21). The p21 protein was shown to have the same function on the tumor necrosis factor (TNF) and tissue plasminogen activator (r-PA) pathways (22). In tumors, p21 inhibits tumor development (23). Low expression of p21 and p53 mutations may play a role in the occurrence of esophageal cancer (24). Esophageal cancer cells show less proliferation after transfection with p21 (25). The cyclin D1 regulatory subunit combines with $\mathrm{CDK}$ s to form functionally active complexes with significant effects on cell proliferation, and overexpression leads to uncontrolled cell growth. Various studies have shown low expression of p21 and high expression of cyclin D1 in esophageal cancer (26-28).

The mechanism of Miz-1 action in esophageal cancer development is still unknown. Studies have shown that high expression of Myc and low regulation of p21 exist in esophageal cancer. Miz-1 has a repressive function on the expression of Myc (29). In the present study, we hypothesized that Miz-1 is a cancer gene acting through binding, or in tumors with high levels of Myc, suppression of downstream genes such as p21 and p15. The expression of Miz-1 in esophageal cancer was determined, and its biological functions were measured after silencing of Miz-1 by shRNA in two esophageal cancer cell lines. Our findings confirmed that Miz-1 acts as a promoting factor and has a vital function in esophageal cancer.

\section{Materials and methods}

Cell lines and tumor samples. A series of esophageal cancer cell lines (Eca109, KYS150, BT9, BT, SEG, Bar-T10 and T9) were used. These cells were obtained from Chongqing Key Laboratory of Molecular Oncology and Epigenetics (Chongqing, China). The cell lines were maintained in RPMI-1640 medium (HyClone Thermo, Beijing, China) supplemented with $10 \%$ fetal bovine serum (FBS; PAN Biotech, Aidenbach, Germany) and $100 \mathrm{U} / \mathrm{ml}$ of penicillin and streptomycin, and grown at $37^{\circ} \mathrm{C}$ in $5 \% \mathrm{CO}_{2}$. DNA and RNA samples were extracted from paired esophageal tumor tissues and para-carcinoma tissues of distal surgical margins. All tissues were obtained from patients who volunteered to receive surgery due to esophageal cancer at the Department of Surgery, The First Affiliated Hospital of Chongqing Medical University. The present study was approved by the Institutional Review Board of Chongqing Medical University.

cDNA and RNA extraction. Total RNA was extracted from the cell lines and tissues using TRIzol reagent (Invitrogen, Waltham, MA, USA), and other basic chemical reagents. Each sample was injected in $1 \mathrm{ml}$ TRIzol. The tissue samples were excised into pieces under liquid nitrogen while using a homogenizer for $5 \mathrm{~min}$, and then $200 \mu \mathrm{l}$ of chloroform was added with mixing. The samples were mixed for $3 \mathrm{~min}$, and the supernatants were obtained after centrifugation for $15 \mathrm{~min}$ at $4^{\circ} \mathrm{C}$ and $16,099 \mathrm{x} \mathrm{g} / \mathrm{min}$. The supernatant samples to be injected $(500 \mu \mathrm{l})$ were kept for $10 \mathrm{~min}$, and then used for further centrifugation. The resulting supernatants were discarded and $1 \mathrm{ml}$ of $75 \%$ ethanol was used for the precipitants, collected after further centrifugation for $5 \mathrm{~min}$ at $4^{\circ} \mathrm{C}$ and $6,288 \times \mathrm{g} / \mathrm{min}$. The clear liquid was removed and diethyl phosphorocyanidate (DEPC) water was injected for blending according to the size of the precipitants. The RT reagent kit (Takara, Dalian, China) was used according to the manufacturer's specification for a $10-\mu 1$ volume system using reverse transcription of RNA into cDNA. The concentration and purity of the cDNA were determined, and samples were stored at $-80^{\circ} \mathrm{C}$ until use.

Semi-quantitative RT-PCR amplification and gel electrophoresis. Real-time reverse transcription polymerase chain reaction (RT-PCR) samples were prepared using the premixed Taq kit (Takara) and amplification was performed on an ABI Prism 7000 detection system (Applied Biosystems, Foster City, CA, USA) according to the conditions recommended by the manufacturer. The $10-\mu 1$ volume system contained $5 \mu 1 \mathrm{Taq}$ polymerase, $3.8 \mu \mathrm{l}$ DEPC, $0.8 \mu \mathrm{l}$ forward and reverse primers and $0.4 \mu \mathrm{l}$ cDNA. The following primers were used: Miz-1 forward, 5'-GTGTGTGATGTGCGGTAAGG-3' and reverse, 5'-GGACTGGACGAATCTCTTGC-3'; p21 forward, 5'-CAG CTGAGGTGTGAGCAGC-3' and reverse, 5'-GACATGGCG CCTCCTCTG-3'; cyclin D1 forward, 5'-GTGTATCGAGAG GCCAAAGG-3' and reverse, 5'-GCAACCAGAAATGCAC AGAC-3'; GAPDH forward, 5'-ACCACCATGGAGAAGG CTGG-3' and reverse, 5'-CTCAGTGTAGCCCAGGATGC-3'. RT-PCR was performed with 30 cycles for Miz-1, cyclin D1 and $\mathrm{p} 21$, and 23 cycles for glyceraldehyde-3-phosphate dehydrogenase (GAPDH). RT-PCR gel electrophoresis used 2\% agarose (Invitrogen, Carlsbad, CA, USA) dissolved in 1X TAE buffer (Double Helix, Shanghai, China). Agarose gel electrophoresis was performed at $120 \mathrm{~mA}$ for $25 \mathrm{~min}$.

Construction and transfection with the lentiviral vector shRNA. Based on the known Miz-1 gene sequence in GenBank (Gene ID: 7709), we commissioned Shanghai Innovation Biotechnology Co. Ltd. to provide the lentiviral vectors which contained Miz-1 shRNA. We chose shRNA in a lentiviral vector due to its stable transfection ability, low cytotoxicity and high efficiency of transfection and gene knockout. The following sequences were packaged into the lentivirus vector: viral, GPH-ZBTB17shRNA-F, GATCCGTGTTCACTTTAAGGCTCATACTTCC TGTCAGATATGAGCCTTAAAGTGAACACTTTTTG and GPH-ZBTB17-shRNA-R, AATTCAAAAAGTGTTCACTTT AAGGCTCATATCTGACAGGAAGTATGAGCCTTAAAGT GAACACG. The cells $\left(1 \times 10^{5}\right)$ were seeded onto 6-well plates, while injecting $20 \mu \mathrm{l}\left(2 \times 10^{8} \mathrm{TU} / \mathrm{ml}\right)$ of the target gene or empty lentiviral vector into each plate. The cell medium was replaced after transfection for $12 \mathrm{~h}$, and fluorescence was observed $72 \mathrm{~h}$ after transfection. The transfected cells were selected with Puromycin (Eca109, $1.2 \mu \mathrm{g} / \mathrm{ml}$; KYS150, $0.8 \mu \mathrm{g} / \mathrm{ml}$ ) on the basis of the experiments using normal cells. After selecting stably transfected cells, the cells were divided into three groups: Miz-1 (transfected with shRNA for knockdown of the Miz-1 gene), vector (transfection with empty viral vectors) and control (normal untreated cells) groups.

Immunohistochemical staining. Immunohistochemistry was performed using the Histostain ${ }^{\circledR}$-Plus kit (Thermo Fisher, 
Waltham, MA, USA). Sections were dewaxed by soaking in xylene for $10 \mathrm{~min}$, four times and then the concentration of ethanol was stepwise decreased from 100 to $75 \%$ for 8 min at every concentration. Endogenous peroxidase was blocked by $3 \%$ hydrogen peroxide for $15 \mathrm{~min}$. Antigen retrieval was carried out using citrate buffer at an elevated but not a boiling temperature for $25 \mathrm{~min}$. The sections were incubated with the primary Miz-1 antibody (1:50 dilution) overnight at $4^{\circ} \mathrm{C}$, followed by secondary antibody incubation at $37^{\circ} \mathrm{C}$ for $30 \mathrm{~min}$. 3,3'-Diaminobenzidine (DAB) was used for staining, and hematoxylin was used for counterstaining. Neutral gum was used to overlay the slides. Phosphate-buffered saline (PBS) was used between each step, for $3 \mathrm{~min}$, three times. The immunohistochemical staining results were assigned a score based on the depth of staining and the rate of dye uptake by the stained cells. The intensity of staining was determined as: 0 , no staining; 1 , weak staining; 2 , moderate staining; and 3 , strong staining. Percentage of positive staining of the cells was determined as: $<5 \%, 0 ; 5-25 \%, 1 ;>25-50 \%, 2 ;>50-75 \%, 3$; and $>75 \%, 4$. Cytoplasmic and nuclear staining was included in the statistical analyses. The index of staining was determined by multiplying the score for the staining intensity by the score for the positive rate. A score of $<4$ was considered as negative staining (marked with a minus sign), and a score $\geq 4$ was considered as positive staining (+).

Cell proliferation assays. Log-phase cells $\left(2 \times 10^{3}\right)$ from three groups were seeded onto 96-well plates. After $24 \mathrm{~h}$, $10 \mu \mathrm{l}$ of Cell Counting Kit-8 (CCK-8; Dojindo Molecular Technologies, Santa Clara, CA, USA) reagent was added into each plate. The optical densities (ODs) were determined using a microplate reader (Infinite $200 \mathrm{PRO}$; Tecan) at $450 \mathrm{~nm}$ after adding CCK- 8 for $2 \mathrm{~h}$. Samples were then likewise measured for 5 days of culture. The experiments were repeated independently three times. The proliferation curve of each group was based on the absorbance at $450 \mathrm{~nm}$.

Colony formation assays. Log-phase cells were seeded onto 6 -well plates with $2 \mathrm{ml}$ of complete medium (500 cells/well) and incubated. Plates were initially examined by microscopy to confirm that only single cells without clumps had been plated. Two weeks after seeding, the colonies were fixed with 4\% paraformaldehyde for $30 \mathrm{~min}$ and stained with crystal violet for $30 \mathrm{~min}$. Images were obtained and counting of the cells was carried out using a scanner.

Migration and invasion assays. Each group of cells ( $4 \times 10^{5}$ cells) was plated into cell culture inserts with $8-\mu \mathrm{m}$ aperture filters (Corning, Corning, NY, USA) coated with (invasion) or without (migration) Matrigel ${ }^{\circledR}$ (Corning) and incubated for $24 \mathrm{~h}$. Cells migrated and invaded from the low to the high concentration of serum. After $24 \mathrm{~h}$, cells passing the filters outside the inserts were fixed in paraformaldehyde, stained with crystal violet, and observed by a microscope. Experiments were performed in triplicate.

Analyses of the cell cycle and apoptosis. Three groups of log-phase cells were collected and centrifuged at $93 \mathrm{x} \mathrm{g} / \mathrm{min}$ for $5 \mathrm{~min}$ after trypsinization. The cells were then washed with PBS twice and fixed in ice-cold $70 \%$ ethanol overnight at $4^{\circ} \mathrm{C}$.
Cells for apoptosis detection were washed with PBS twice and mixed in PBS in 1-ml Eppendorf tubes. The samples were assayed for cell cycle and apoptosis at Chongqing Medical University Life Sciences Institute.

Western blot analyses. Cells with stable transfection or wild-type were collected by a cell wiper and washed with PBS twice. Cell proteins were extracted by one mixture of 99\% RIPA lysis buffer (RIPA) and 1\% phenylmethanesulfonyl fluoride (PMSF) (Beytime Biotechnology, Shanghai, China) according to the cell mass weight after centrifugation at $4^{\circ} \mathrm{C}$ for $40 \mathrm{~min}$, then centrifuged at $4^{\circ} \mathrm{C}$, at $12,000 \mathrm{rpm}$ for $15 \mathrm{~min}$. After centrifugation, loading buffer was added at a volume of $25 \%$ of the liquid supernatant and samples were boiled for $5 \mathrm{~min}$. A total of $40 \mu \mathrm{g}$ of protein was separated by sodium dodecyl sulphate-polyacrylamide gel electrophoresis (SDS-PAGE) and then transferred onto a polyvinylidene fluoride (PVDF) membrane using the laboratory manual instructions. Membranes were blocked in 5\% skimmed milk powder for $2 \mathrm{~h}$. The primary antibodies used were: Miz-1 (Santa Cruz Biotechnology, Santa Cruz, CA, USA), p21, cleaved caspase 3, polyADP ribose polymerase (PARP) (all from Cell Signaling Technology, Danvers, MA, USA), cyclin D1 (Epitomics, Burlingame, CA, USA) and GAPDH used as a control (Cell Signaling Technology). Membranes were incubated with the primary antibodies at $4^{\circ} \mathrm{C}$ overnight, washed with Tris-buffered saline and Tween-20 (TBST) twice, and immersed in the corresponding secondary antibodies for 2 h. Proteins were visualized by BeyoECL Plus (Beyotime, Chengdu, China) in the dark.

Statistical analyses. All statistical analyses were performed with SPSS 17.0 software (SPSS, Inc., Chicago, IL, USA). Data are expressed as the average \pm standard deviation (SD). Student's t-tests were used for comparisons between any two means and single factor analyses of variance (ANOVA) were used for comparisons among more than two groups. The Chi-square test was used for two constituent ratios in two different groups. For all values, $\mathrm{p}<0.05$ was considered to indicate a statistically significant result. All graphs were created by GraphPad Prism (GraphPad, La Jolla, CA, USA).

\section{Results}

Miz-1 is highly expressed in esophageal cancer and cell lines. We collected 22 esophageal tumor tissues and paired distal surgical margin samples from normal esophageal tissues to examine the expression of Miz-1. Half of the samples were used for RT-PCR and the other half for immunohistochemical staining. We found that expression of Miz-1 was upregulated in 8 tumor tissue samples compared with that in the normal esophageal tissues, as determined by RT-PCR (Fig. 1C). Similar results were found using immunohistochemical staining. Nine of 11 samples were positive (Fig. 1D) in the group of tumor tissues; however, three samples were positive in the control samples. We also detected Miz-1 expression in 7 esophageal cancer cell lines, by RT-PCR. The results showed that Miz-1 was expressed at high levels in the esophageal cancer cell lines (Eca109 and KYS150) (Fig. 1B). The results suggest that Miz-1 is an oncogene in esophageal cancer. 
A

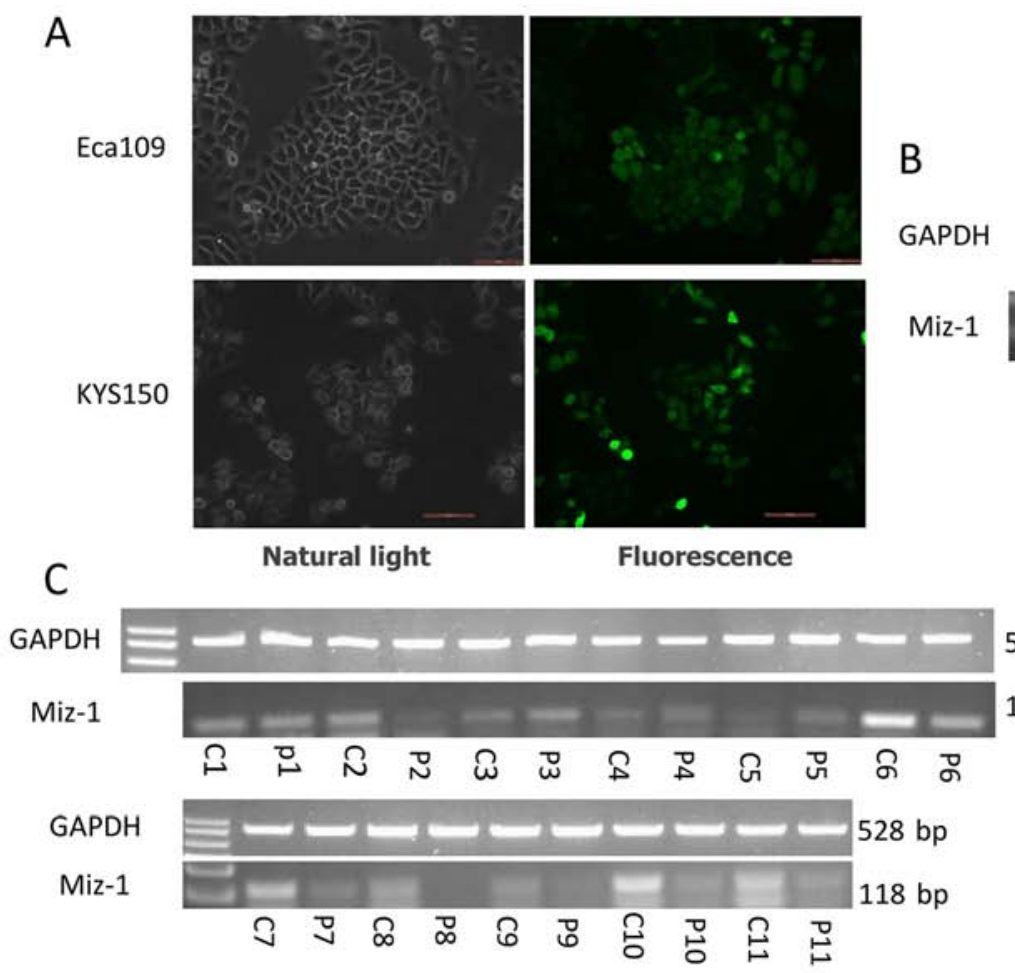

D

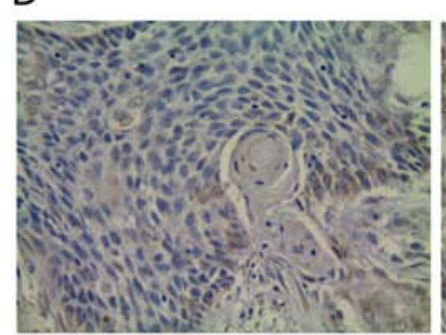

Juxtacancerous

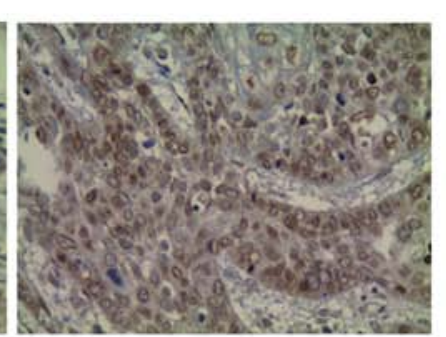

Carcinoma

\begin{tabular}{llll}
\hline & \multicolumn{2}{c}{ Expression } & \\
\cline { 2 - 3 } \multicolumn{1}{c}{ Type } & + & - & p-value \\
\hline Juxtacancerous & 3 & 8 & 0.03 \\
Carcinoma & 9 & 2 & \\
\hline
\end{tabular}

Note: Statistical analysis: Chi-square test; -: negative

(score of 0-4); +: positive (score of 5-12). $\mathrm{p}<0.05$.

Figure 1. Expression of Miz-1 in esophageal cancer cell lines, transfected cells, esophageal cancer tissues and juxtacancerous tissues. (A) Fluorescence images show high transfection efficiency of the shRNA lentivirus in two esophageal cancer cell lines (Eca109 and KYS150) (photomicrographs are shown at a magnification of x400). (B) Expression of Miz-1 in 7 esophageal cancer cell lines. We chose the two highest expressing cell lines Eca109 and KYS150. (C) Miz-1 in 11 paired cancer $(\mathrm{C})$ and juxtacancerous tissues $(\mathrm{P})$. Seven of the esophageal cancer tissues had a higher level of Miz-1 than that in the adjacent cancer tissues. Paired t-test was used for statistical analysis $(\mathrm{p}<0.05)$. (D) Immunohistochemical assay revealed the same outcome; Miz-1 was upregulated in most cancer tissues compared with that in the distant tissues (photomicrographs are shown at a magnification of $\mathrm{x} 400$ ). Chi-square test was used for statistics ( $\mathrm{p}<0.05$ ).

Transfection verification. The green fluorescence-containing shRNA lentivirus showed the Miz-1 (transfected with shRNA to knockout the Miz-1 gene) and the vector (transfected with the empty viral vector) cell groups by use of fluorescence microscopy. After stable transfection, Miz-1 mRNA levels were measured by RT-PCR and the protein expression of Miz-1 was measured by western blot analyses. We confirmed that cells were successfully transfected with the lentivirus by the presence of green fluorescence (Fig. 1A). We also further confirmed Miz-1 knockdown in the cells by RT-PCR and western blot analyses (Fig. 4A and B).

Miz-1 promotes cell clonogenicity and proliferation. After we confirmed that Miz-1 is differentially expression in tumor tissues vs. normal tissues, we determined whether the cell functions were altered after knockdown of Miz-1. The colony formation and CCK- 8 assays were used to determine the cell proliferation status. The results showed that the colony formation numbers (104 in Eca109 and 65 in KYS150) in the group with silenced Miz-1 was markedly reduced compared to that in the control group ( $<<0.05)$ (Fig. 2B). Similarly, the CCK-8 assay showed inhibition of cell growth in the two cell lines after Miz-1 was silenced ( $\mathrm{p}<0.05$ ) (Fig. 2A).

Miz-1 reduces cell cycle arrest and apoptosis. We further investigated the effect of Miz-1 on esophageal cancer cell apoptosis and the cell cycle using flow cytometry. The Miz-1 cell group with knockdown of Miz-1 had increased numbers of Eca109 (60.8\%) and KYS150 (57\%) cells in the G0-G1 phase compared with the control cells $(\mathrm{p}<0.05)$ (Fig. 2C). Furthermore, knockdown of Miz-1 induced cell apoptosis from 9.3 to $28.6 \%$ in the Eca109 cells and from 16.8 to $24.7 \%$ in the KYS150 cells $(\mathrm{p}<0.05)$ (Fig. 3C). These results suggest that the cell cycle and apoptosis were also affected by Miz-1. Moreover, knockdown of Miz-1 induced apoptosis.

Miz-1 induces invasiveness and migration. Cellular motility through invasiveness and migration experiments was further 

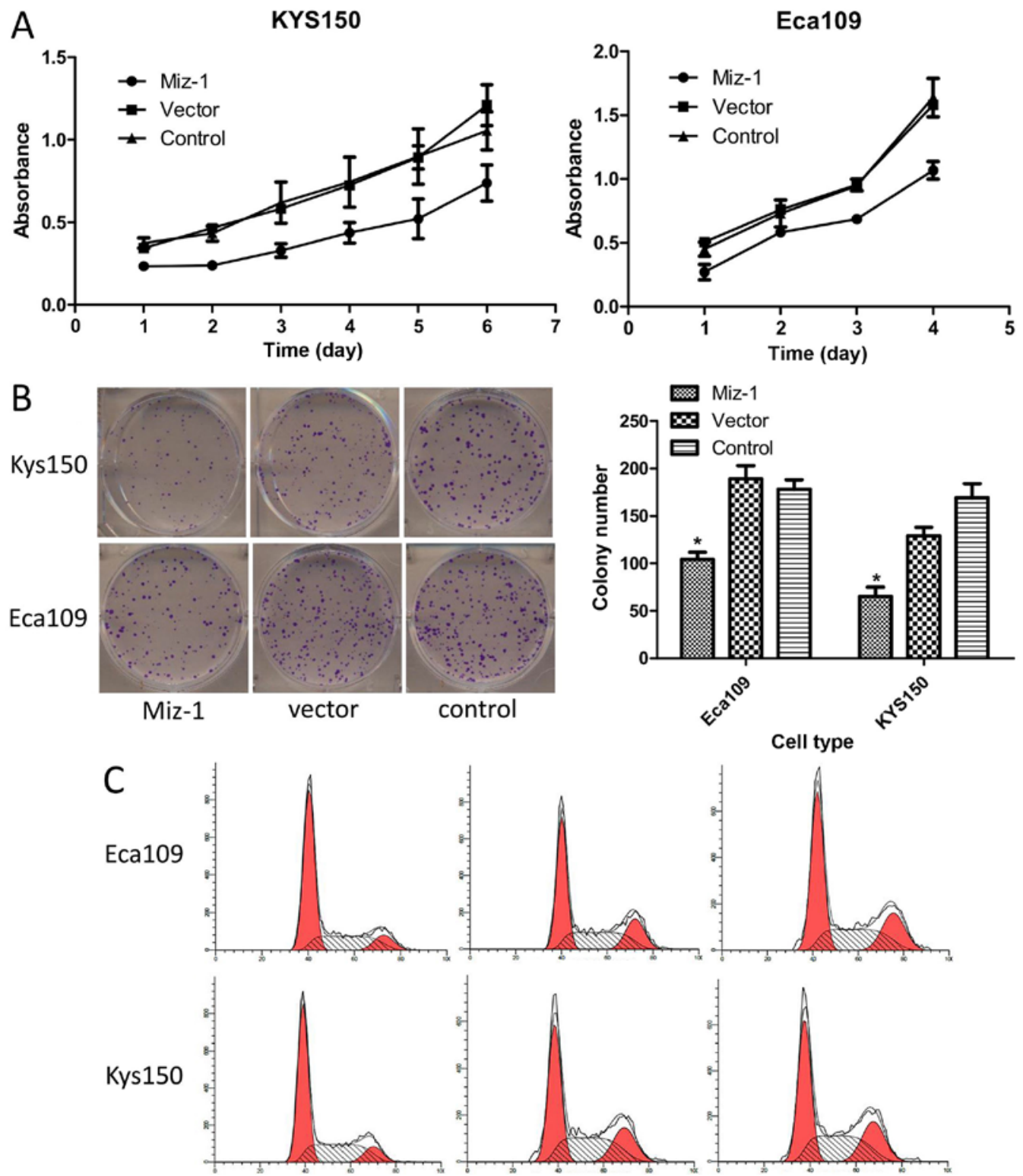

Miz-1
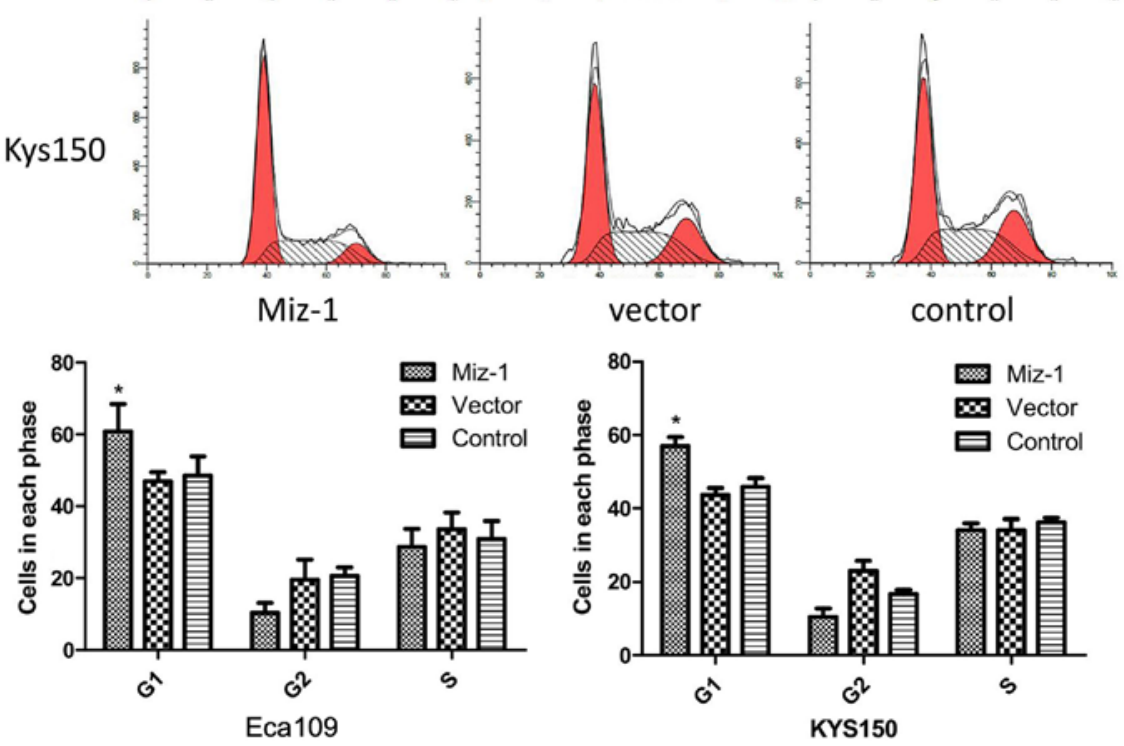

Figure 2. Effect of Miz-1 on the proliferation and cell cycle distribution in two types of esophageal cancer cell lines (Eca109 and KYS150). (A) The CCK-8 proliferation assay showed that knockdown of Miz-1 inhibited the cell proliferation rates of the Eca109 and KYS150 cells (p<0.05). (B) Colony formation assay. Miz-1-shRNA-transfected Eca109 and KYS105 cells formed fewer colonies compared with the colony number in the empty vector and control cells $(\mathrm{p}<0.05)$ (original magnification, $\mathrm{x} 40)$. (C) The cell cycle was analyzed using flow cytometry in Eca109 and KYS150 cells. The Miz-1 shRNA-knockdown group had a higher proportion of cells arrested in the G0/G1 phase $(\mathrm{p}<0.05)$. Eca109 cells were also arrested in the G1/G2 phase ( $<<0.05)$, but the KYS150 cells were not $(\mathrm{p}>0.05)$. There was no difference in the proliferation, colony formation or cell cycle distribution between the empty vector and control cells in the two cell lines $(\mathrm{p}>0.05)$.

determined. The cells transfected with the Miz-1 shRNA had a low capability for invasiveness and migration, using both
Eca109 and KYS150 cells (Fig. 3A and B). In the absence of a matrix, the number of Eca109 cells with Miz-1 knock- 
A

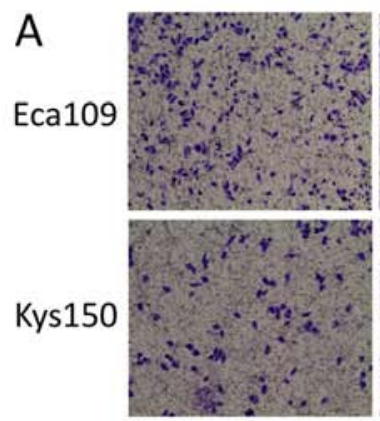

Miz-1

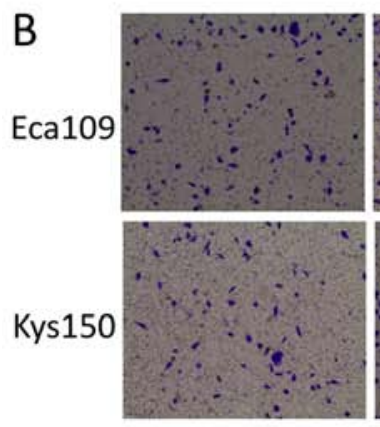

Miz-1
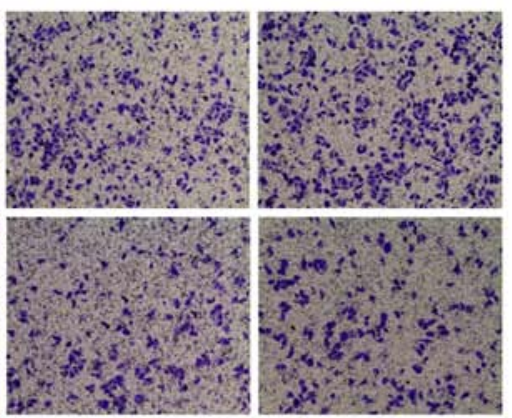

vector

control
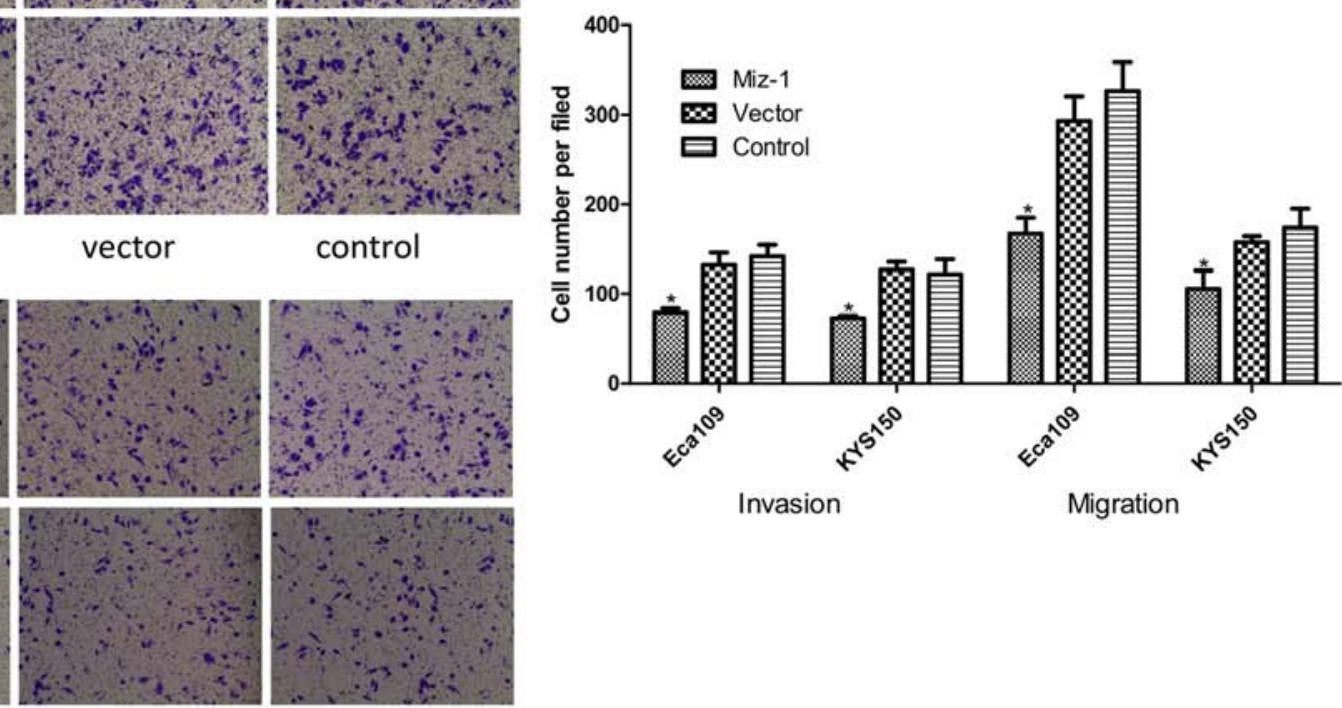

control
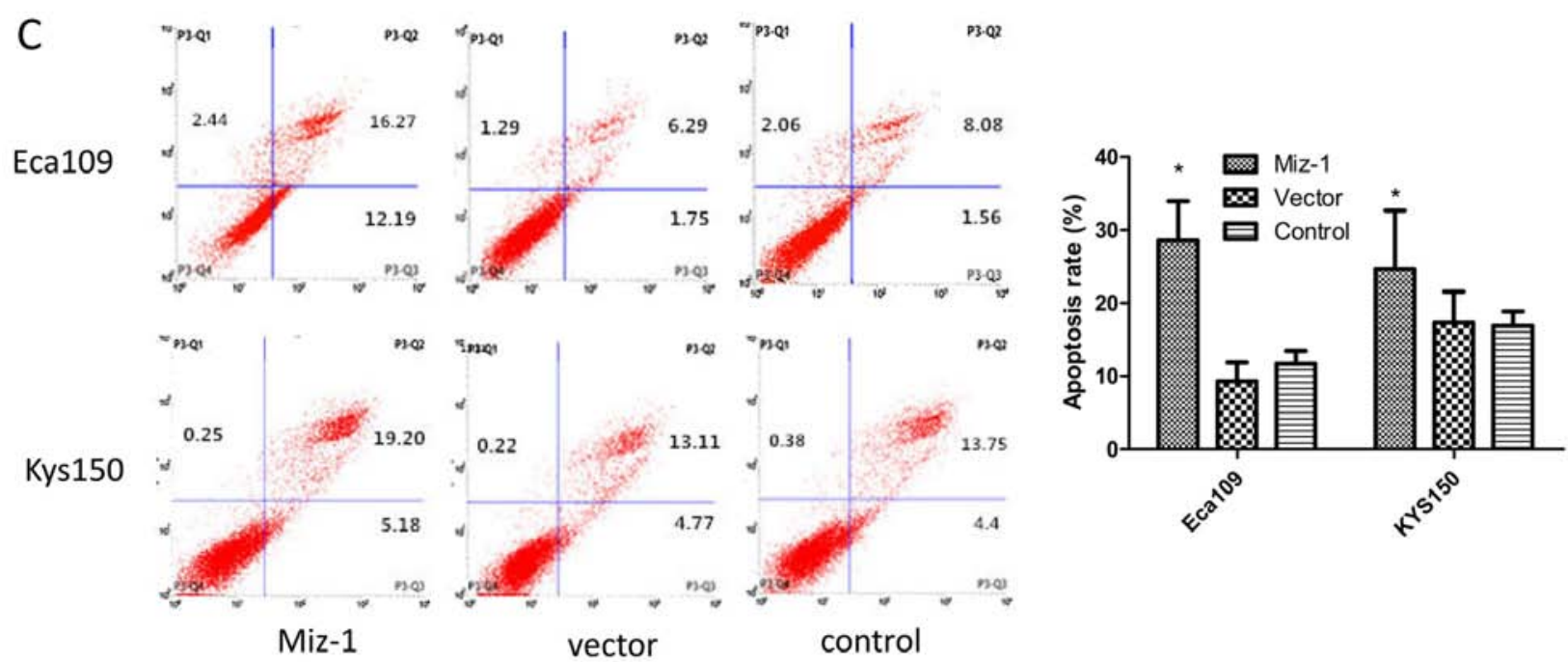

Figure 3. Influence of Miz-1 on the apoptosis, migration and invasion in the Eca109 and KYS150 cells. (A) The migration assay showed that knockdown of Miz-1 inhibited the migration ability in the Eca109 and KYS150 cells (p<0.05). (B) The same results occurred in the invasion assay (p<0.05) (photomicrographs are shown at a magnification of x100). (C) Induction of apoptosis as detected by flow cytometry with Annexin V-PE staining. The cell group without target gene Miz-1 had a higher rate of apoptosis compared with other two control groups ( $\mathrm{p}>0.05)$.

down (168 cells) passing through the Transwell was less than the control cells (293 knockdown and 326 control cells) in five microscopic fields $(\mathrm{p}<0.05)$. Similar results were also observed for the invasiveness assays using the KYS150 cell line $(\mathrm{p}<0.05)$.

Miz-1 decreases the expression of p21, caspase 3, PARP and p21-arrested cyclin D1. We also examined Miz-1 as a potential downstream target gene using RT-PCR and western blot analyses. We hypothesized that Miz-1 could be a link between the c-Myc oncogene and the downstream target genes p21 and cyclin D1. The results suggested that without Miz-1, p21 was expressed at high levels and reduced downstream cyclin D1, leading to cell cycle arrest (Fig. 4A and B). Moreover, knockdown of Miz-1 induced apoptotic factors caspase 3 and PARP (Fig. 4A and B) $(\mathrm{p}<0.05)$.

\section{Discussion}

Esophageal cancer is one of the most common cancers. It originates in the esophageal mucosal epithelium. Cell cycle dysregulation is one fundamental aspect of cancer incidence and development. The proliferation of cancer cells is attributed to unchecked cell cycling (32), leading to the lack of cell death and perhaps to cell pleomorphism. The p21 is one inhibitor of CDK that monitors abnormal proliferation in cells. In esophageal cancer, there is a low level of p21, and cell cycle dysregulation. The cause may be a mutation of p21 itself, or 

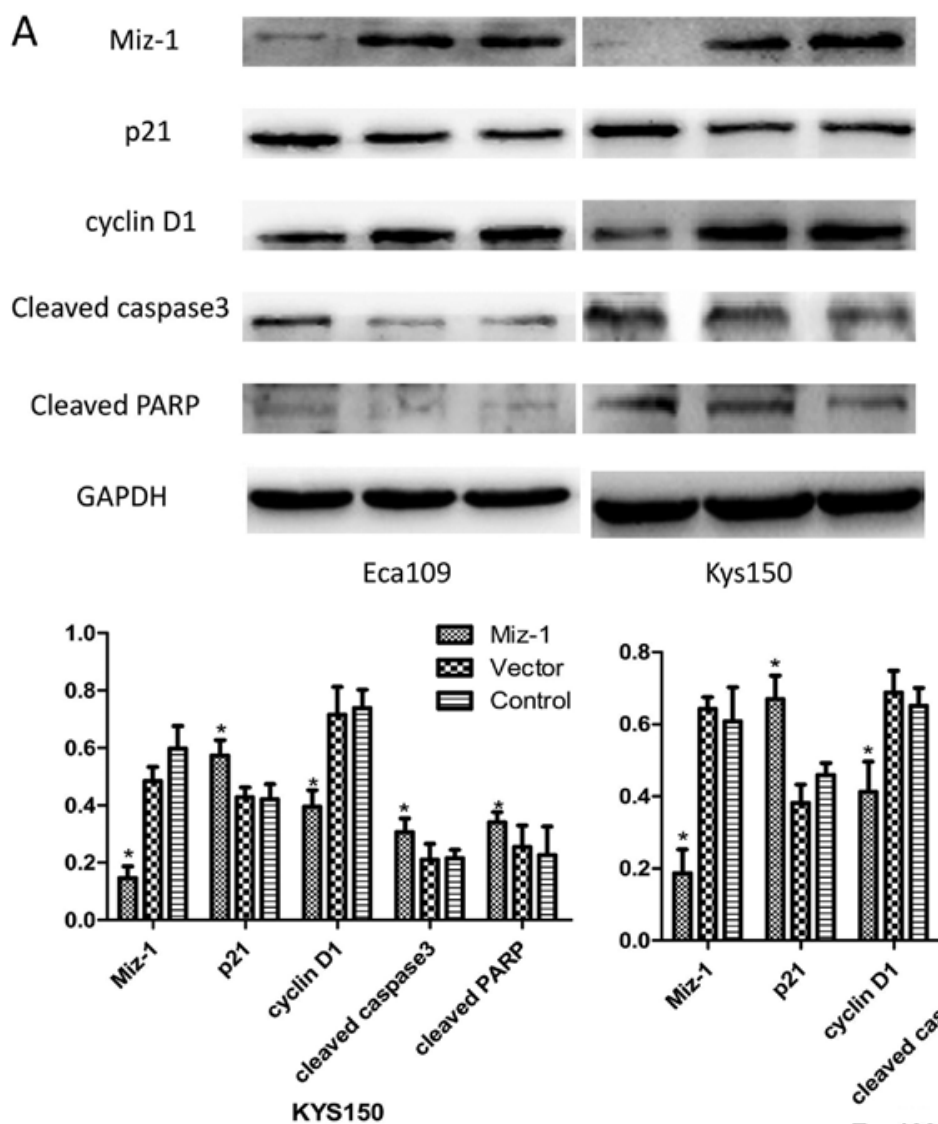

$19 \mathrm{kDa}$

$89 \mathrm{kDa}$
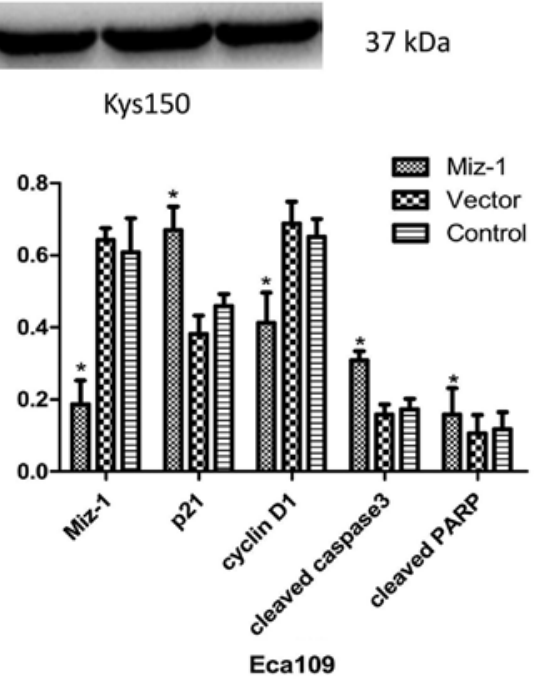

B

Miz-1

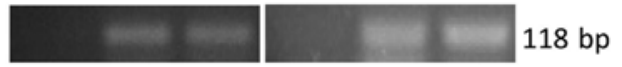

P21

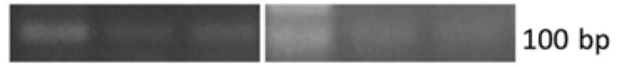

cyclin D1
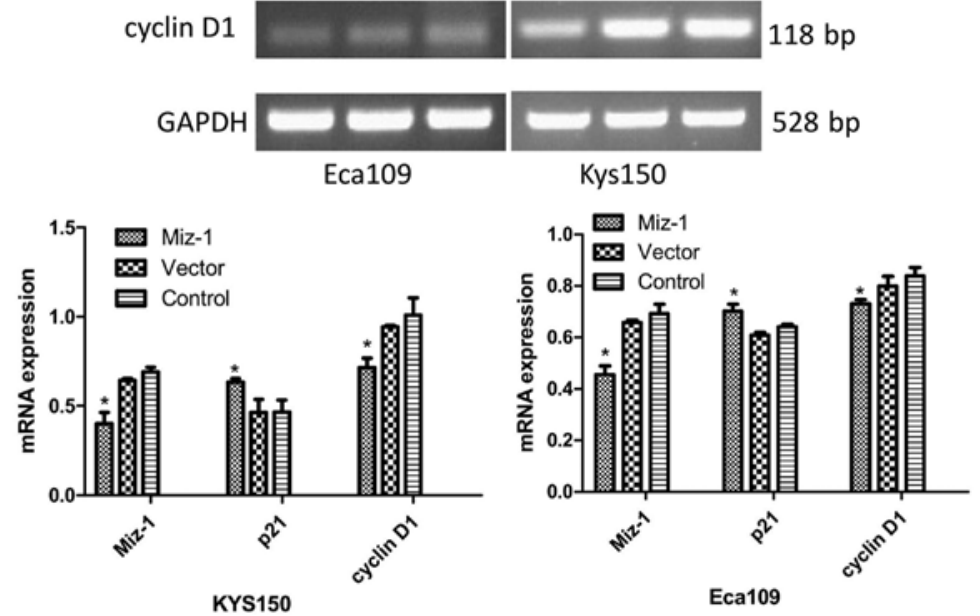

Figure 4. Expression of proteins in three groups of cells with knockdown of Miz-1, the empty vector and control. (A) Western blot assay verified the efficiency of target gene Miz-1 ( $\mathrm{p}<0.05)$. Proteins of its downstream also changed following knockdown of Miz-1 ( $<<0.05)$. Another effect was the classical pathway of apoptosis. (B) The same results were confirmed by RT-PCR $(\mathrm{p}<0.05)$. Vector and control groups showed similar protein levels $(\mathrm{p}>0.05)$.

the loss of regulation of upstream c-Myc oncogenes. Several studies have shown high levels of c-Myc in esophageal cancer. We hypothesized that this cell cycle signaling pathway may be critical for esophageal cancer development.

To determine the pathway of oncogenesis, we showed that Miz-1 one zinc finger protein interaction between Myc was necessary for inhibition of $\mathrm{p} 21$ by Myc. To further confirm these findings, we used two stable esophageal cancer cell lines with Miz-1 silencing via specific shRNA packaged in a lentiviral vector. Fluorescence microscopy images confirmed the success of the transfection. The RT-PCR and western blot analyses verified the Miz-1 downregulation in the transfected 
cell lines. Using the same methodology, we measured the expression levels of both p21 and cyclin D1.

The most obvious changes in the cell cycle and proliferation were observed in the Miz-1-silenced cells. Likewise, cyclin D1 restored the inhibition of $\mathrm{p} 21$. Flow cytometric results suggested that the cells with knockdown of Miz-1 remained in G0-G1 phase, even in the presence of cyclin D1, a factor that maintains cells in the G1-S phase. Since the cell cycle was arrested in the G1 phase, the cells were smaller and had less proliferation. Our studies revealed that the cell group with downregulated Miz-1 had less growth and proliferation compared with the other cell groups as determined by the CCK-8 and clone formation assays.

Decreasing Miz-1 not only had an effect on the cell cycle and proliferation, but also influenced apoptosis and cell migration. The migration and invasion assays showed that the cells moved more slowly after knockdown of the target gene. The data also suggested that Miz-1 participated in apoptotic signaling pathways through altered expression of PARP and caspase 3 . The results of the cell apoptosis experiments suggested that cells without Miz-1 had a higher rate of apoptosis compared with the other two cell groups.

Our findings are consistent with the hypothesis that Miz-1 is a key regulator in the signaling pathway of cell cycle arrest induced by CDKN1a and cyclin D1. Moreover, we found that Miz-1 also participated in apoptosis through caspase 3 and PARP.

Overall, our results strongly suggest that Miz-1 is highly expressed in esophageal cancer tissues compared with juxtacancerous tissues (at least $5 \mathrm{~cm}$ distance from the tumor). This suggested that esophageal cancer may develop when the levels of Miz-1 are elevated. When we knocked down Miz-1, we found changes in cell proliferation, in the cell cycle distribution, apoptosis, migration and invasion. We therefore propose a signaling pathway by which Miz-1 suppresses p21, a normal cancer suppressor gene that regulates the cell cycle. One possible mechanism is that Miz-1 acts with some oncogene such as Myc. Miz-1 could also function independently; however, its mechanism remains unknown.

We chose to measure two typical apoptosis-related proteins in the classic signaling pathways. The alteration of caspase 3 and PARP demonstrated that Miz-1 participates in apoptotic signaling pathways. The migration and invasion of cells are attributed to the functions of integrins or the influences of cell viability and cell cycle arrest. To confirm these results, further coimmunoprecipitation assays and/or dual-luciferase reporter systems should be used to confirm a relationship between these two target genes.

Our findings are consistent with the hypothesis that Miz-1 has a different expression pattern in tumors vs. juxta cancerous tissues and may act as a key regulator of oncogenesis by affecting various cell functions, including the cell cycle and apoptosis. These findings indicate that Miz-1 may be an effective and novel target for esophageal cancer therapy.

\section{Acknowledgements}

The present study was supported by the Key Scientific Research Project of Chongqing Municipal Bureau of Health (grant no. 2012-1-015).

\section{References}

1. Dang CV: c-Myc target genes involved in cell growth, apoptosis, and metabolism. Mol Cell Biol 19: 1-11, 1999.

2. Wu Y and Li Z: Oncogene c-myc and malignant tumor. Clin Res 25: 1698-1700, 2008 .

3. Kotake T, Saiki S, Kinouchi T, Shiku H and Nakayama E: Detection of the c-myc gene product in urinary bladder cancer. Jpn J Cancer Res 81: 1198-1201, 1990.

4. Masters JR, Vesey SG, Munn CF, Evan GI and Watson JV: c-myc oncoprotein levels in bladder cancer. Urol Res 16: 341-344, 1988.

5. Levens D: Disentangling the MYC web. Proc Natl Acad Sci USA 99: 5757-5759, 2002.

6. Li LH, Nerlov C, Prendergast G, MacGregor D and Ziff EB: c-Myc represses transcription in vivo by a novel mechanism dependent on the initiator element and Myc box II. EMBO J 13: 4070-4079, 1994.

7. Peukert K, Staller P, Schneider A, Carmichael G, Hänel F and Eilers M: An alternative pathway for gene regulation by Myc. EMBO J 16: 5672-5686, 1997.

8. Phan RT, Saito M, Basso K, Niu H and Dalla-Favera R: BCL6 interacts with the transcription factor Miz-1 to suppress the cyclin-dependent kinase inhibitor p21 and cell cycle arrest in germinal center B cells. Nat Immunol 6: 1054-1060, 2005.

9. Basu S, Liu Q, Qiu Y and Dong F: Gfi-1 represses CDKN2B encoding $\mathrm{p} 15^{\mathrm{INK} 4 \mathrm{~B}}$ through interaction with Miz-1. Proc Natl Acad Sci USA 106: 1433-1438, 2009.

10. Lu J, Chen M, Ren XR, Wang J, Lyerly HK, Barak L and Chen W: Regulation of Hedgehog signaling by Myc-interacting zinc finger protein 1, Miz1. PLoS One 8: e63353, 2013.

11. Inoue S, Hao Z, Elia AJ, Cescon D, Zhou L, Silvester J, Snow B, Harris IS, Sasaki M, Li WY, et al: Mule/Huwe1/Arf-BP1 suppresses Ras-driven tumorigenesis by preventing c-Myc/Miz1mediated down-regulation of p21 and p15. Genes Dev 27: 1101-1114, 2013.

12. van Riggelen J, Müller J, Otto T, Beuger V, Yetil A, Choi PS, Kosan C, Möröy T, Felsher DW and Eilers M: The interaction between Myc and Miz1 is required to antagonize TGFbetadependent autocrine signaling during lymphoma formation and maintenance. Genes Dev 24: 1281-1294, 2010.

13. Gebhardt A, Kosan C, Herkert B, Möröy T, Lutz W, Eilers M and Elsässer HP: Mizl is required for hair follicle structure and hair morphogenesis. J Cell Sci 120: 2586-2593, 2007.

14. Seoane J, Le HV and Massagué J: Myc suppression of the $p 21^{\text {Cipl }}$ Cdk inhibitor influences the outcome of the p53 response to DNA damage. Nature 419: 729-734, 2002.

15. Wu S, Cetinkaya C, Munoz-Alonso MJ, von der Lehr N, Bahram F, Beuger V, Eilers M, Leon J and Larsson LG: Myc represses differentiation-induced $p 21 C I P 1$ expression via Miz-1-dependent interaction with the $p 21$ core promoter. Oncogene 22: 351-360, 2003.

16. Adhikary S, Peukert K, Karsunky H, Beuger V, Lutz W, Elsässer HP, Möröy T and Eilers M: Mizl is required for early embryonic development during gastrulation. Mol Cell Biol 23: 7648-7657, 2003

17. Gebhardt A, Frye M, Herold S, Benitah SA, Braun K, Samans B, Watt FM, Elsässer HP and Eilers M: Myc regulates keratinocyte adhesion and differentiation via complex formation with Miz1. J Cell Biol 172: 139-149, 2006.

18. Saba I, Kosan C, Vassen L and Möröy T: IL-7R-dependent survival and differentiation of early T-lineage progenitors is regulated by the $\mathrm{BTB} / \mathrm{POZ}$ domain transcription factor Miz-1. Blood 117: 3370-3381, 2011.

19. Hönnemann J, Sanz-Moreno A, Wolf E, Eilers M and Elsässer HP: Mizl is a critical repressor of $c d k n l a$ during skin tumorigenesis. PLoS One 7: e34885, 2012

20. Liu J, Yan J, Jiang S, Wen J, Chen L, Zhao Y and Lin A: Sitespecific ubiquitination is required for relieving the transcription factor Miz1-mediated suppression on TNF- $\alpha$-induced JNK activation and inflammation. Proc Natl Acad Sci USA 109: 191-196, 2012.

21. Li Z and Li B: Correlation of gene polymorphism of p21 and p27 with tumor. Int J Gene 29: 317-320, 2006.

22. Gerhard Krauss (Translated by: Sun C,Liu JS, et al): Biochemistry of Signal Transduction and Regulation. Chemical Industry Press, pp338-377, 2005

23. Palazzo JP, Mercer WE, Kovatich AJ and McHugh M: Immunohistochemical localization of $\mathrm{p} 21^{\mathrm{WAF} / \mathrm{CIP} 1}$ in normal, hyperplastic, and neoplastic uterine tissues. Hum Pathol 28: 60-66, 1997. 
24. Zhang J, Lv H, Jing L and Qin J: Expression of p21 $1^{\text {cip } 1 / \text { WAF }}$ p53 and the infection of HPV-16 in the esophageal cancer. Shi Yong Ai Zheng Za Zhi She 19: 488-490, 2004 (In Chinese).

25. Tanaka Y, Fujii T, Yamana H, Kato S, Morimatsu M and Shirouzu $\mathrm{K}$ : Experimental gene therapy using p $21^{\text {Wafl }}$ gene for esophageal squamous cell carcinoma by gene gun technology. Int J Mol Med 14: 545-551, 2004

26. Liu J, Hu Y, Hu W, Xie X, Ela Bella A,Fu J and Rao D: Expression and prognostic relevance of $\mathrm{p} 21^{\mathrm{WAF} 1}$ in stage III esophageal squamous cell carcinoma. Dis Esophagus 25: 67-71, 2012.

27. Zhang H, Liu Y and $\mathrm{Hu} \mathrm{M}$ : Expression and significance of p53, p21, p16, cyclin D and CDK, in esophageal squamous cell carcinoma. Immunological J 4: 312-315, 2003.

28. Möröy T, Saba I and Kosan C: The role of the transcription factor Miz-1 in lymphocyte development and lymphomagenesis-binding Myc makes the difference. Semin Immunol 23: 379-387, 2011.
29. Montgomery EA: Oesophageal cancer. In: World Cancer Report 2014. Stewart BW and Wild CP (eds). International Agency for Research on Cancer, Lyon, pp374-382, 2014.

30. Jemal A, Bray F, Center MM, Ferlay J, Ward E and Forman D: Global cancer statistics. CA Cancer J Clin 61: 69-90, 2011.

31. Chen W, Zheng R, Zhang S, Zhao P, Zeng H and Zou X: Report of cancer incidence and mortality in China, 2010. Ann Transl Med 2: 61, 2014

32. Deshpande A, Sicinski P and Hinds PW: Cyclins and cdks in development and cancer: A perspective. Oncogene 24: 2909-2915, 2005. 